Filomat xx (yyyy), zzz-zzz

DOI (will be added later)
Published by Faculty of Sciences and Mathematics, University of Niš, Serbia

Available at: http://www.pmf.ni.ac.rs/filomat

\title{
Some Cosmological Solutions of a Nonlocal Modified Gravity
}

\author{
Ivan Dimitrijevic ${ }^{\mathrm{a}}$, Branko Dragovich ${ }^{\mathrm{b}}$, Jelena Grujic ${ }^{\mathrm{c}}$, Zoran Rakic ${ }^{\mathrm{a}}$ \\ ${ }^{a}$ Faculty of Mathematics, University of Belgrade, Studentski trg 16, Belgrade, Serbia \\ ${ }^{b}$ Institute of Physics, University of Belgrade, Pregrevica 118, 11080 Belgrade, Serbia \\ ${ }^{c}$ Teacher Education Faculty, University of Belgrade, Kraljice Natalije 43, Belgrade, Serbia
}

\begin{abstract}
We consider nonlocal modification of the Einstein theory of gravity in framework of the pseudoRiemannian geometry. The nonlocal term has the form $\mathcal{H}(R) \mathcal{F}(\square) \mathcal{G}(R)$, where $\mathcal{H}$ and $\mathcal{G}$ are differentiable functions of the scalar curvature $R$, and $\mathcal{F}(\square)=\sum_{n=0}^{\infty} f_{n} \square^{n}$ is an analytic function of the d'Alambert operator ․ Using calculus of variations of the action functional, we derived the corresponding equations of motion. The variation of action is induced by variation of the gravitational field, which is the metric tensor $g_{\mu v}$. Cosmological solutions are found for the case when the Ricci scalar $R$ is constant.
\end{abstract}

\section{Introduction}

Although very successful, Einstein theory of gravity is not a final theory. There are many its modifications, which are motivated by quantum gravity, string theory, astrophysics and cosmology (for a review, see [1]). One of very promising directions of research is nonlocal modified gravity and its applications to cosmology (as a review, see [2, 3] and [4]). To solve cosmological Big Bang singularity, nonlocal gravity with replacement $R \rightarrow R+C R \mathcal{F}(\square) R$ in the Einstein-Hilbert action was proposed in [5]. This nonlocal model is further elaborated in the series of papers [6-12].

In [13] we introduced a new approach to nonlocal gravity given by the action

$$
S=\int d^{4} x \sqrt{-g}\left(\frac{R}{16 \pi G}+R^{-1} \mathcal{F}(\square) R\right)
$$

where the $\mathrm{d}^{\prime}$ Alembert operator is $\square=\frac{1}{\sqrt{-g}} \partial_{\mu} \sqrt{-g} g^{\mu v} \partial_{\nu}, g=\operatorname{det}\left(g_{\mu v}\right)$. The nonlocal term $R^{-1} \mathcal{F}(\square) R=$ $f_{0}+R^{-1} \sum_{n=1}^{\infty} f_{n} \square^{n} R$ contains $f_{0}$ which can be connected with the cosmological constant as $f_{0}=-\frac{\Lambda}{8 \pi G}$. This term is also invariant under transformation $R \rightarrow C R$, where $C$ is a constant, i.e. this nonlocality does not depend on magnitude of the scalar curvature $R \neq 0$.

2010 Mathematics Subject Classification. 83D05, 53B21, 53B50; Secondary 53C25, 83F05

Keywords. nonlocal gravity, cosmological solutions, equations of motion, calculus of variations, pseudo-Riemannian manifold.

Received: dd Month yyyy; Accepted: dd Month yyyy

Communicated by (name of the Editor, mandatory)

Work on this paper was supported by Ministry of Education, Science and Technological Development of the Republic of Serbia, grant No 174012.

Email addresses: ivand@matf.bg.ac.rs (Ivan Dimitrijevic), dragovich@ipb.ac.rs (Branko Dragovich), jelenagg@gmail.com (Jelena Grujic), zrakic@matf.bg.ac.rs (Zoran Rakic) 
In this paper we consider $n$-dimensional pseudo-Riemannian manifold $M$ with metric $g_{\mu v}$ of signature $\left(n_{-}, n_{+}\right)$. Our nonlocal gravity model here is larger than (1) and given by the action

$$
S=\int_{M}\left(\frac{R-2 \Lambda}{16 \pi G}+\mathcal{H}(R) \mathcal{F}(\square) \mathcal{G}(R)\right) \sqrt{|g|} \mathrm{d}^{n} x,
$$

which is a functional of metric (gravitational field) $g_{\mu \nu}$, where $\mathcal{H}$ and $\mathcal{G}$ are differentiable functions of the scalar curvature $R$, and $\Lambda$ is cosmological constant.

\section{Variation of the action functional}

Let us introduce the following auxiliary functionals

$$
S_{0}=\int_{M}(R-2 \Lambda) \sqrt{|g|} \mathrm{d}^{n} x, \quad S_{1}=\int_{M} \mathcal{H}(R) \mathcal{F}(\square) \mathcal{G}(R) \sqrt{|g|} \mathrm{d}^{n} x .
$$

Then the variations of $S_{0}$ and $S_{1}$ can be considered separately and the variation of (2) can be expressed as

$$
\delta S=\frac{1}{16 \pi G} \delta S_{0}+\delta S_{1}
$$

Note that variations of the metric tensor elements and their first derivatives are zero on the boundary of manifold $M$, i.e. $\left.\delta g_{\mu \nu}\right|_{\partial M}=0,\left.\delta \partial_{\lambda} g_{\mu \nu}\right|_{\partial M}=0$.

Lemma 2.1. Let $M$ be a pseudo-Riemannian manifold. Then the following basic relations hold:

$$
\begin{aligned}
\frac{\partial g^{\mu v}}{\partial x^{\sigma}} & =-g^{\mu \alpha} \Gamma_{\sigma \alpha}^{v}-g^{v \alpha} \Gamma_{\sigma \alpha,}^{\mu} & \delta g & =g g^{\mu v} \delta g_{\mu v}=-g g_{\mu v} \delta g^{\mu v}, \\
\Gamma_{\mu v}^{\mu} & =\frac{\partial}{\partial x^{v}} \ln \sqrt{|g|}, & \delta \sqrt{|g|} & =-\frac{1}{2} g_{\mu v} \sqrt{|g|} \delta g^{\mu v}, \\
\square & =\nabla^{\mu} \nabla_{\mu}=\frac{1}{\sqrt{|g|}} \partial_{\mu}\left(\sqrt{|g|} g^{\mu v} \partial_{v}\right), & \delta R & =R_{\mu v} \delta g^{\mu v}+g_{\mu v} \square \delta g^{\mu v}-\nabla_{\mu} \nabla_{\nu} \delta g^{\mu v} .
\end{aligned}
$$

Lemma 2.2. On the manifold $M$ holds $\int_{M} g^{\mu v} \delta R_{\mu v} \sqrt{|g|} \mathrm{d}^{n} x=0$.

Proof. Let $W^{v}=-g^{\mu \alpha} \delta \Gamma_{\mu \alpha}^{v}+g^{\mu v} \delta \Gamma_{\mu \alpha}^{\alpha}$. Then it follows

$$
\frac{1}{\sqrt{|g|}} \frac{\partial}{\partial x^{v}}\left(\sqrt{|g|} W^{v}\right)=\frac{\partial W^{v}}{\partial x^{v}}+W^{v} \frac{1}{\sqrt{|g|}} \frac{\partial \sqrt{|g|}}{\partial x^{v}} .
$$

Using Lemma 2.1 we get

$$
\begin{aligned}
\frac{1}{\sqrt{|g|}} \frac{\partial}{\partial x^{v}}\left(\sqrt{|g|} W^{v}\right) & =-\frac{\partial}{\partial x^{v}}\left(g^{\mu \alpha} \delta \Gamma_{\mu \alpha}^{v}\right)+\frac{\partial}{\partial x^{v}}\left(g^{\mu v} \delta \Gamma_{\mu \alpha}^{\alpha}\right)+\left(-g^{\mu \alpha} \delta \Gamma_{\mu \alpha}^{v}+g^{\mu v} \delta \Gamma_{\mu \alpha}^{\alpha}\right) \Gamma_{\nu \beta}^{\beta} \\
& =-\frac{\partial g^{\mu \alpha}}{\partial x^{v}} \delta \Gamma_{\mu \alpha}^{v}-g^{\mu \alpha} \delta \frac{\partial \Gamma_{\mu \alpha}^{v}}{\partial x^{v}}+\frac{\partial g^{\mu v}}{\partial x^{v}} \delta \Gamma_{\mu \alpha}^{\alpha}+g^{\mu v} \delta \frac{\partial \Gamma_{\mu \alpha}^{\alpha}}{\partial x^{v}}+\left(-g^{\mu \alpha} \delta \Gamma_{\mu \alpha}^{v}+g^{\mu v} \delta \Gamma_{\mu \alpha}^{\alpha}\right) \Gamma_{\nu \beta}^{\beta} .
\end{aligned}
$$


Moreover, using again Lemma2.1 we obtain

$$
\begin{aligned}
\frac{1}{\sqrt{|g|}} \frac{\partial}{\partial x^{v}}\left(\sqrt{|g|} W^{v}\right) & =g^{\alpha \beta} \Gamma_{\nu \beta}^{\mu} \delta \Gamma_{\mu \alpha}^{v}+g^{\mu \beta} \Gamma_{\nu \beta}^{\alpha} \delta \Gamma_{\mu \alpha}^{v}-g^{\beta v} \Gamma_{\nu \beta}^{\mu} \delta \Gamma_{\mu \alpha}^{\alpha}-g^{\mu \beta} \Gamma_{\nu \beta}^{v} \delta \Gamma_{\mu \alpha}^{\alpha}-g^{\mu \alpha} \delta \frac{\partial \Gamma_{\mu \alpha}^{v}}{\partial x^{v}}+g^{\mu v} \delta \frac{\partial \Gamma_{\mu \alpha}^{\alpha}}{\partial x^{v}} \\
& -g^{\mu \alpha} \Gamma_{\nu \beta}^{\beta} \delta \Gamma_{\mu \alpha}^{v}+g^{\mu v} \Gamma_{\nu \beta}^{\beta} \delta \Gamma_{\mu \alpha}^{\alpha} \\
& =g^{\mu v}\left(-\delta \frac{\partial \Gamma_{\mu v}^{\alpha}}{\partial x^{\alpha}}+\delta \frac{\partial \Gamma_{\mu \alpha}^{\alpha}}{\partial x^{v}}+\Gamma_{\alpha \mu}^{\beta} \delta \Gamma_{\beta v}^{\alpha}+\Gamma_{\nu \beta}^{\alpha} \delta \Gamma_{\mu \alpha}^{\beta}-\Gamma_{v \mu}^{\beta} \delta \Gamma_{\beta \alpha}^{\alpha}-\Gamma_{\beta \alpha}^{\alpha} \delta \Gamma_{\mu v}^{\beta}\right) \\
& =g^{\mu v} \delta\left(-\frac{\partial \Gamma_{\mu v}^{\alpha}}{\partial x^{\alpha}}+\frac{\partial \Gamma_{\mu \alpha}^{\alpha}}{\partial x^{v}}+\Gamma_{\alpha \mu}^{\beta} \Gamma_{\beta v}^{\alpha}-\Gamma_{\beta \alpha}^{\alpha} \Gamma_{\mu v}^{\beta}\right)=g^{\mu v} \delta R_{\mu v} .
\end{aligned}
$$

Finally, we have

$$
g^{\mu v} \delta R_{\mu v}=\frac{1}{\sqrt{|g|}} \frac{\partial}{\partial x^{v}}\left(\sqrt{|g|} W^{v}\right) \text { and } \int_{M} g^{\mu v} \delta R_{\mu v} \sqrt{|g|} \mathrm{d}^{n} x=\int_{M} \frac{\partial}{\partial x^{v}}\left(\sqrt{|g|} W^{v}\right) \mathrm{d}^{n} x .
$$

Using the Gauss-Stokes theorem one obtains

$$
\int_{M} \frac{\partial}{\partial x^{v}}\left(\sqrt{|g|} W^{v}\right) \mathrm{d}^{n} x=\int_{\partial M} W^{v} d \sigma_{v}
$$

Since $\delta g_{\mu v}=0$ and $\delta \partial_{\lambda} g_{\mu v}=0$ at the boundary $\partial M$ we have $\left.W^{v}\right|_{\partial M}=0$. Then we have $\int_{\partial M} W^{v} d \sigma_{v}=0$, that completes the proof.

Lemma 2.3. The variation of $S_{0}$ is

$$
\delta S_{0}=\int_{M} G_{\mu v} \sqrt{|g|} \delta g^{\mu v} \mathrm{~d}^{n} x+\Lambda \int_{M} g_{\mu v} \sqrt{|g|} \delta g^{\mu v} \mathrm{~d}^{n} x,
$$

where $G_{\mu v}=R_{\mu v}-\frac{1}{2} R g_{\mu v}$ is the Einstein tensor.

Proof. The variation of $S_{0}$ can be found as follows

$$
\begin{aligned}
\delta S_{0} & =\int_{M} \delta((R-2 \Lambda) \sqrt{|g|}) \mathrm{d}^{n} x=\int_{M} \delta(R \sqrt{|g|}) \mathrm{d}^{n} x-2 \Lambda \int_{M} \delta \sqrt{|g|} \mathrm{d}^{n} x \\
& =\int_{M}\left(\sqrt{|g|} \delta R+R \delta \sqrt{|g|}+\Lambda g_{\mu v} \sqrt{|g|} \delta g^{\mu v}\right) \mathrm{d}^{n} x \\
& =\int_{M}\left(\sqrt{|g|} \delta\left(g^{\mu v} R_{\mu v}\right)-\frac{1}{2} R \sqrt{|g|} g_{\mu v} \delta g^{\mu v}+\Lambda g_{\mu v} \sqrt{|g|} \delta g^{\mu v}\right) \mathrm{d}^{n} x \\
& =\int_{M} G_{\mu v} \sqrt{|g|} \delta g^{\mu v} \mathrm{~d}^{n} x+\Lambda \int_{M} g_{\mu v} \sqrt{|g|} \delta g^{\mu v} \mathrm{~d}^{n} x+\int_{M} g^{\mu v} \delta R_{\mu v} \sqrt{|g|} \mathrm{d}^{n} x .
\end{aligned}
$$

Using Lemma 2.2, from the last equation we obtain the variation of $S_{0}$.

Lemma 2.4. For any scalar function $h$ we have

$$
\int_{M} h \delta R \sqrt{|g|} \mathrm{d}^{n} x=\int_{M}\left(h R_{\mu v}+g_{\mu v} \square h-\nabla_{\mu} \nabla_{v} h\right) \delta g^{\mu \nu} \sqrt{|g|} \mathrm{d}^{n} x .
$$

Proof. Using Lemma 2.1, for any scalar function $h$ we have

$$
\int_{M} h \delta R \sqrt{|g|} \mathrm{d}^{n} x=\int_{M}\left(h R_{\mu v} \delta g^{\mu v}+h g_{\mu v} \square \delta g^{\mu v}-h \nabla_{\mu} \nabla_{\nu} \delta g^{\mu v}\right) \sqrt{|g|} \mathrm{d}^{n} x .
$$


The second and third term in this formula can be transformed in the following way:

$$
\begin{aligned}
& \int_{M} h g_{\mu v}\left(\square \delta g^{\mu v}\right) \sqrt{|g|} \mathrm{d}^{n} x=\int_{M} g_{\mu v}(\square h) \delta g^{\mu v} \sqrt{|g|} \mathrm{d}^{n} x, \\
& \int_{M} h \nabla_{\mu} \nabla_{v} \delta g^{\mu v} \sqrt{|g|} \mathrm{d}^{n} x=\int_{M} \nabla_{\mu} \nabla_{v} h \delta g^{\mu v} \sqrt{|g|} \mathrm{d}^{n} x .
\end{aligned}
$$

To prove the first of these equations we use the Stokes theorem and obtain

$$
\begin{aligned}
\int_{M} h g_{\mu \nu} \square \delta g^{\mu v} \sqrt{|g|} \mathrm{d}^{n} x & =\int_{M} h g_{\mu \nu} \nabla_{\alpha} \nabla^{\alpha} \delta g^{\mu v} \sqrt{|g|} \mathrm{d}^{n} x=-\int_{M} \nabla_{\alpha}\left(h g_{\mu v}\right) \nabla^{\alpha} \delta g^{\mu v} \sqrt{|g|} \mathrm{d}^{n} x \\
& =\int_{M} g_{\mu \nu} \nabla^{\alpha} \nabla_{\alpha} h \delta g^{\mu v} \sqrt{|g|} \mathrm{d}^{n} x=\int_{M} g_{\mu \nu} \square h \delta g^{\mu v} \sqrt{|g|} \mathrm{d}^{n} x .
\end{aligned}
$$

Here we have used $\nabla_{\gamma} g_{\mu \nu}=0$ and $\nabla^{\alpha} \nabla_{\alpha}=\nabla_{\alpha} \nabla^{\alpha}=\square$ to obtain the last integral.

To obtain the second equation we first introduce vector

$$
N^{\mu}=h \nabla_{v} \delta g^{\mu \nu}-\nabla_{\nu} h \delta g^{\mu \nu}
$$

From the above expression follows

$$
\begin{aligned}
\nabla_{\mu} N^{\mu} & =\nabla_{\mu}\left(h \nabla_{v} \delta g^{\mu v}-\nabla_{v} h \delta g^{\mu v}\right)=\nabla_{\mu} h \nabla_{v} \delta g^{\mu v}+h \nabla_{\mu} \nabla_{v} \delta g^{\mu v}-\nabla_{\mu} \nabla_{v} h \delta g^{\mu v}-\nabla_{v} h \nabla_{\mu} \delta g^{\mu v} \\
& =h \nabla_{\mu} \nabla_{v} \delta g^{\mu v}-\nabla_{\mu} \nabla_{v} h \delta g^{\mu v} .
\end{aligned}
$$

Integrating $\nabla_{\mu} N^{\mu}$ yields $\int_{M} \nabla_{\mu} N^{\mu} \sqrt{|g|} \mathrm{d}^{n} x=\int_{\partial M} N^{\mu} n_{\mu} \mathrm{d} \partial M$, where $n_{\mu}$ is the unit normal vector. Since $\left.N^{\mu}\right|_{\partial M}=0$ we have that the last integral is zero, which completes the proof.

Lemma 2.5. Let $\theta$ and $\psi$ be scalar functions such that $\left.\delta \psi\right|_{\partial M}=0$. Then one has

$$
\begin{aligned}
\int_{M} \theta \delta \square \psi \sqrt{|g|} \mathrm{d}^{n} x & =\frac{1}{2} \int_{M} g^{\alpha \beta} \partial_{\alpha} \theta \partial_{\beta} \psi g_{\mu \nu} \delta g^{\mu \nu} \sqrt{|g|} \mathrm{d}^{n} x-\int_{M} \partial_{\mu} \theta \partial_{\nu} \psi \delta g^{\mu v} \sqrt{|g|} \mathrm{d}^{n} x \\
& +\int_{M} \square \theta \delta \psi \sqrt{|g|} \mathrm{d}^{n} x+\frac{1}{2} \int_{M} g_{\mu \nu} \theta \square \psi \delta g^{\mu \nu} \sqrt{|g|} \mathrm{d}^{n} x .
\end{aligned}
$$

Proof. Since $\theta$ and $\psi$ are scalar functions such that $\left.\delta \psi\right|_{\partial M}=0$ we have

$$
\begin{aligned}
& \int_{M} \theta \delta \square \psi \sqrt{|g|} \mathrm{d}^{n} x=\int_{M} \theta \partial_{\alpha} \delta\left(\sqrt{|g|} g^{\alpha \beta} \partial_{\beta} \psi\right) \mathrm{d}^{n} x+\int_{M} \theta \delta\left(\frac{1}{\sqrt{|g|}}\right) \partial_{\alpha}\left(\sqrt{|g|} g^{\alpha \beta} \partial_{\beta} \psi\right) \sqrt{|g|} \mathrm{d}^{n} x \\
& =\int_{M} \partial_{\alpha}\left(\theta \delta\left(\sqrt{|g|} g^{\alpha \beta} \partial_{\beta} \psi\right)\right) \mathrm{d}^{n} x-\int_{M} \partial_{\alpha} \theta \delta\left(\sqrt{|g|} g^{\alpha \beta} \partial_{\beta} \psi\right) \mathrm{d}^{n} x+\frac{1}{2} \int_{M} \theta g_{\mu v} \square \psi \delta g^{\mu \nu} \sqrt{|g|} \mathrm{d}^{n} x .
\end{aligned}
$$


It is easy to see that $\int_{M} \partial_{\alpha}\left(\theta \delta\left(\sqrt{|g|} g^{\alpha \beta} \partial_{\beta} \psi\right)\right) \mathrm{d}^{n} x=0$. From this result it follows

$$
\begin{aligned}
& \int_{M} \theta \delta \square \psi \sqrt{|g|} \mathrm{d}^{n} x=-\int_{M} g^{\alpha \beta} \partial_{\alpha} \theta \partial_{\beta} \psi \delta(\sqrt{|g|}) \mathrm{d}^{n} x-\int_{M} \partial_{\alpha} \theta \partial_{\beta} \psi \delta g^{\alpha \beta} \sqrt{|g|} \mathrm{d}^{n} x \\
& -\int_{M} g^{\alpha \beta} \sqrt{|g|} \partial_{\alpha} \theta \partial_{\beta} \delta \psi \mathrm{d}^{n} x+\frac{1}{2} \int_{M} \theta g_{\mu v} \square \psi \delta g^{\mu \nu} \sqrt{|g|} \mathrm{d}^{n} x \\
& =\frac{1}{2} \int_{M} g^{\alpha \beta} \partial_{\alpha} \theta \partial_{\beta} \psi g_{\mu \nu} \delta g^{\mu \nu} \sqrt{|g|} \mathrm{d}^{n} x-\int_{M} \partial_{\mu} \theta \partial_{\nu} \psi \delta g^{\mu v} \sqrt{|g|} \mathrm{d}^{n} x \\
& -\int_{M} \partial_{\beta}\left(g^{\alpha \beta} \sqrt{|g|} \partial_{\alpha} \theta \delta \psi\right) \mathrm{d}^{n} x+\int_{M} \partial_{\beta}\left(g^{\alpha \beta} \sqrt{|g|} \partial_{\alpha} \theta\right) \delta \psi \mathrm{d}^{n} x \\
& +\frac{1}{2} \int_{M} g_{\mu \nu} \theta \square \psi \delta g^{\mu v} \sqrt{|g|} \mathrm{d}^{n} x \\
& =\frac{1}{2} \int_{M} g^{\alpha \beta} \partial_{\alpha} \theta \partial_{\beta} \psi g_{\mu \nu} \delta g^{\mu v} \sqrt{|g|} \mathrm{d}^{n} x-\int_{M} \partial_{\mu} \theta \partial_{\nu} \psi \delta g^{\mu \nu} \sqrt{|g|} \mathrm{d}^{n} x \\
& +\int_{M} \square \theta \delta \psi \sqrt{|g|} \mathrm{d}^{n} x+\frac{1}{2} \int_{M} g_{\mu \nu} \theta \square \psi \delta g^{\mu v} \sqrt{|g|} \mathrm{d}^{n} x .
\end{aligned}
$$

At the end we have that

$$
\begin{aligned}
& \int_{M} \theta \delta \square \psi \sqrt{|g|} \mathrm{d}^{n} x=\frac{1}{2} \int_{M} g^{\alpha \beta} \partial_{\alpha} \theta \partial_{\beta} \psi g_{\mu \nu} \delta g^{\mu \nu} \sqrt{|g|} \mathrm{d}^{n} x \\
& -\int_{M} \partial_{\mu} \theta \partial_{\nu} \psi \delta g^{\mu v} \sqrt{|g|} \mathrm{d}^{n} x+\int_{M} \square \theta \delta \psi \sqrt{|g|} \mathrm{d}^{n} x+\frac{1}{2} \int_{M} g_{\mu \nu} \theta \square \psi \delta g^{\mu v} \sqrt{|g|} \mathrm{d}^{n} x .
\end{aligned}
$$

Now, after this preliminary work we can get the variation of $S_{1}$.

Lemma 2.6. The variation of $S_{1}$ is

$$
\begin{aligned}
\delta S_{1} & =-\frac{1}{2} \int_{M} g_{\mu v} \mathcal{H}(R) \mathcal{F}(\square) \mathcal{G}(R) \delta g^{\mu v} \sqrt{|g|} \mathrm{d}^{n} x+\int_{M}\left(R_{\mu v} \Phi-K_{\mu v} \Phi\right) \delta g^{\mu v} \sqrt{|g|} \mathrm{d}^{n} x \\
& +\frac{1}{2} \sum_{n=1}^{\infty} f_{n} \sum_{l=0}^{n-1} \int_{M}\left(g_{\mu v}\left(\partial^{\alpha} \square^{l} \mathcal{H}(R) \partial_{\alpha} \square^{n-1-l} \mathcal{G}(R)+\square^{l} \mathcal{H}(R) \square^{n-l} \mathcal{G}(R)\right)\right. \\
& \left.-2 \partial_{\mu} \square^{l} \mathcal{H}(R) \partial_{\nu} \square^{n-1-l} \mathcal{G}(R)\right) \delta g^{\mu v} \sqrt{|g|} \mathrm{d}^{n} x,
\end{aligned}
$$

where $K_{\mu v}=\nabla_{\mu} \nabla_{v}-g_{\mu v} \square, \Phi=\mathcal{H}^{\prime}(R) \mathcal{F}(\square) \mathcal{G}(R)+\mathcal{G}^{\prime}(R) \mathcal{F}(\square) \mathcal{H}(R)$ and denotes derivative with respect to $R$.

Proof. The variation of $S_{1}$ can be expressed as

$$
\delta S_{1}=\int_{M}(\mathcal{H}(R) \mathcal{F}(\square) \mathcal{G}(R) \delta(\sqrt{|g|})+\delta(\mathcal{H}(R)) \mathcal{F}(\square) \mathcal{G}(R) \sqrt{|g|}+\mathcal{H}(R) \delta(\mathcal{F}(\square) \mathcal{G}(R)) \sqrt{|g|}) \mathrm{d}^{n} x .
$$

For the first two integrals in the last equation we have

$$
\begin{aligned}
& I_{1}=\int_{M} \mathcal{H}(R) \mathcal{F}(\square) \mathcal{G}(R) \delta(\sqrt{|g|}) \mathrm{d}^{n} x=-\frac{1}{2} \int_{M} g_{\mu \nu} \mathcal{H}(R) \mathcal{F}(\square) \mathcal{G}(R) \delta g^{\mu v} \sqrt{|g|} \mathrm{d}^{n} x, \\
& I_{2}=\int_{M} \delta(\mathcal{H}(R)) \mathcal{F}(\square) \mathcal{G}(R) \sqrt{|g|} \mathrm{d}^{n} x=\int_{M} \mathcal{H}^{\prime}(R) \delta R \mathcal{F}(\square) \mathcal{G}(R) \sqrt{|g|} \mathrm{d}^{n} x .
\end{aligned}
$$


Substituting $h=\mathcal{H}^{\prime}(R) \mathcal{F}(\square) \mathcal{G}(R)$ in equation (12) we obtain

$$
I_{2}=\int_{M}\left(R_{\mu v} \mathcal{H}^{\prime}(R) \mathcal{F}(\square) \mathcal{G}(R)-K_{\mu v}\left(\mathcal{H}^{\prime}(R) \mathcal{F}(\square) \mathcal{G}(R)\right)\right) \delta g^{\mu v} \sqrt{|g|} \mathrm{d}^{n} x .
$$

The third integral can be presented using linear combination of the following integrals

$$
J_{n}=\int_{M} \mathcal{H}(R) \delta\left(\square^{n} \mathcal{G}(R)\right) \sqrt{|g|} \mathrm{d}^{n} x .
$$

$J_{0}$ is the integral of the same form as $I_{2}$ so

$$
J_{0}=\int_{M}\left(R_{\mu v} \mathcal{G}^{\prime}(R) \mathcal{H}(R)-K_{\mu v}\left(\mathcal{G}^{\prime}(R) \mathcal{H}(R)\right)\right) \delta g^{\mu v} \sqrt{|g|} \mathrm{d}^{n} x
$$

For $n>0$, we can find $J_{n}$ using (19). In the first step we take $\theta=\mathcal{H}(R)$ and $\psi=\square^{n-1} \mathcal{G}(R)$ and obtain

$$
\begin{aligned}
J_{n} & =\frac{1}{2} \int_{M} g^{\alpha \beta} \partial_{\alpha} \mathcal{H}(R) \partial_{\beta} \square^{n-1} \mathcal{G}(R) g_{\mu \nu} \delta g^{\mu \nu} \sqrt{|g|} \mathrm{d}^{n} x-\int_{M} \partial_{\mu} \mathcal{H}(R) \partial_{\nu} \square^{n-1} \mathcal{G}(R) \delta g^{\mu v} \sqrt{|g|} \mathrm{d}^{n} x \\
& +\int_{M} \square \mathcal{H}(R) \delta \square^{n-1} \mathcal{G}(R) \sqrt{|g|} \mathrm{d}^{n} x+\frac{1}{2} \int_{M} g_{\mu \nu} \mathcal{H}(R) \square^{n} \mathcal{G}(R) \delta g^{\mu v} \sqrt{|g|} \mathrm{d}^{n} x .
\end{aligned}
$$

In the second step we take $\theta=\square \mathcal{H}(R)$ and $\psi=\square^{n-2} \mathcal{G}(R)$ and get the third integral in this formula, etc. Using (19) $n$ times one obtains

$$
\begin{aligned}
J_{n} & =\frac{1}{2} \sum_{l=0}^{n-1} \int_{M}\left(g_{\mu \nu} \partial^{\alpha} \square^{l} \mathcal{H}(R) \partial_{\alpha} \square^{n-1-l} \mathcal{G}(R)+g_{\mu v} \square^{l} \mathcal{H}(R) \square^{n-l} \mathcal{G}(R)-2 \partial_{\mu} \square^{l} \mathcal{H}(R) \partial_{\nu} \square^{n-1-l} \mathcal{G}(R)\right) \delta g^{\mu v} \sqrt{|g|} \mathrm{d}^{n} x \\
& +\int_{M}\left(R_{\mu v} \mathcal{G}^{\prime}(R) \square^{n} \mathcal{H}(R)-K_{\mu v}\left(\mathcal{G}^{\prime}(R) \square^{n} \mathcal{H}(R)\right)\right) \delta g^{\mu v} \sqrt{|g|} \mathrm{d}^{n} x
\end{aligned}
$$

Using the equation (12) we obtain the last integral in the above formula. Finally, one can put everything together and obtain

$$
\begin{aligned}
\delta S_{1} & =I_{1}+I_{2}+\sum_{n=0}^{\infty} f_{n} J_{n}=-\frac{1}{2} \int_{M} g_{\mu v} \mathcal{H}(R) \mathcal{F}(\square) \mathcal{G}(R) \delta g^{\mu v} \sqrt{|g|} \mathrm{d}^{n} x+\int_{M}\left(R_{\mu v} \Phi-K_{\mu v} \Phi\right) \delta g^{\mu v} \sqrt{|g|} \mathrm{d}^{n} x \\
& +\frac{1}{2} \sum_{n=1}^{\infty} f_{n} \sum_{l=0}^{n-1} \int_{M}\left(g_{\mu v}\left(\partial^{\alpha} \square^{l} \mathcal{H}(R) \partial_{\alpha} \square^{n-1-l} \mathcal{G}(R)+\square^{l} \mathcal{H}(R) \square^{n-l} \mathcal{G}(R)\right)\right. \\
& \left.-2 \partial_{\mu} \square^{l} \mathcal{H}(R) \partial_{\nu} \square^{n-1-l} \mathcal{G}(R)\right) \delta g^{\mu v} \sqrt{|g|} \mathrm{d}^{n} x .
\end{aligned}
$$

Theorem 2.1. The variation of the functional (2) is equal to zero iff

$$
\begin{aligned}
& \frac{G_{\mu v}+\Lambda g_{\mu v}}{16 \pi G}-\frac{1}{2} g_{\mu v} \mathcal{H}(R) \mathcal{F}(\square) \mathcal{G}(R)+\left(R_{\mu v} \Phi-K_{\mu v} \Phi\right) \\
& +\frac{1}{2} \sum_{n=1}^{\infty} f_{n} \sum_{l=0}^{n-1}\left(g_{\mu \nu} \partial^{\alpha} \square^{l} \mathcal{H}(R) \partial_{\alpha} \square^{n-1-l} \mathcal{G}(R)-2 \partial_{\mu} \square^{l} \mathcal{H}(R) \partial_{\nu} \square^{n-1-l} \mathcal{G}(R)+g_{\mu v} \square^{l} \mathcal{H}(R) \square^{n-l} \mathcal{G}(R)\right)=0 .
\end{aligned}
$$

Proof. Since we have $\delta S=\frac{1}{16 \pi \mathrm{G}} \delta S_{0}+\delta S_{1}$ the theorem follows from Lemmas 2.3 and 2.6. 


\section{Signature $(1,3)$}

In the physics settings, where functional $S$ represents an action, theorem 2.1 gives the equations of motion. From this point we assume that manifold $M$ is the four-dimensional homogeneous and isotropic one with signature $(1,3)$. Then the metric has the Friedmann-Lemaitre-Robertson-Walker (FLRW) form:

$$
\mathrm{d} s^{2}=-\mathrm{d} t^{2}+a(t)^{2}\left(\frac{\mathrm{d} r^{2}}{1-k r^{2}}+r^{2} \mathrm{~d} \theta^{2}+r^{2} \sin ^{2} \theta \mathrm{d} \varphi^{2}\right) .
$$

Theorem 3.1. Suppose that manifold $M$ has the FLRW metric. Then the expression (31) has two linearly independent equations:

$$
\begin{aligned}
& \frac{4 \Lambda-R}{16 \pi G}-2 \mathcal{H}(R) \mathcal{F}(\square) \mathcal{G}(R)+(R \Phi+3 \square \Phi) \\
& +\sum_{n=1}^{\infty} f_{n} \sum_{l=0}^{n-1}\left(\partial^{\mu} \square^{l} \mathcal{H}(R) \partial_{\mu} \square^{n-1-l} \mathcal{G}(R)+2 \square^{l} \mathcal{H}(R) \square^{n-l} \mathcal{G}(R)\right)=0, \\
& \frac{G_{00}+\Lambda g_{00}}{16 \pi G}-\frac{1}{2} g_{00} \mathcal{H}(R) \mathcal{F}(\square) \mathcal{G}(R)+\left(R_{00} \Phi-K_{00} \Phi\right) \\
& +\frac{1}{2} \sum_{n=1}^{\infty} f_{n} \sum_{l=0}^{n-1}\left(g_{00} \partial^{\alpha} \square^{l} \mathcal{H}(R) \partial_{\alpha} \square^{n-1-l} \mathcal{G}(R)-2 \partial_{0} \square^{l} \mathcal{H}(R) \partial_{0} \square^{n-1-l} \mathcal{G}(R)+g_{00} \square^{l} \mathcal{H}(R) \square^{n-l} \mathcal{G}(R)\right)=0 .
\end{aligned}
$$

Proof. The FLRW metric satisfies $R_{\mu v}=\frac{R}{4} g_{\mu v}$ and scalar curvature $R=6\left(\frac{\ddot{a}}{a}+\left(\frac{\dot{a}}{a}\right)^{2}+\frac{k}{a^{2}}\right)$ depends only on $t$, hence equations (31) for $\mu \neq v$ are trivially satisfied. On the other hand, equations with indices 11, 22 and 33 can be rewritten as

$$
g_{\mu \mu}\left(\frac{-\frac{R}{4}+\Lambda}{8 \pi G}-\mathcal{H}(R) \mathcal{F}(\square) \mathcal{G}(R)+\frac{R}{2} \Phi+\sum_{n=1}^{\infty} f_{n} \sum_{l=0}^{n-1}\left(\partial^{\alpha} \square^{l} \mathcal{H}(R) \partial_{\alpha} \square^{n-1-l} \mathcal{G}(R)+\square^{l} \mathcal{H}(R) \square^{n-l} \mathcal{G}(R)\right)\right)=0 .
$$

Therefore these three equations are linearly dependent and there are only two linearly independent equations. The most convenient choice is the trace and 00-equation.

Corollary 3.1. For $\mathcal{H}(R)=R^{p}$ and $\mathcal{G}(R)=R^{q}$ the action (2) becomes

$$
S=\int_{M}\left(\frac{R-2 \Lambda}{16 \pi G}+R^{p} \mathcal{F}(\square) R^{q}\right) \sqrt{|g|} \mathrm{d}^{n} x,
$$

and equations of motion are

$$
\begin{aligned}
& \frac{1}{16 \pi G}\left(G_{\mu \nu}+\Lambda g_{\mu v}\right)-\frac{1}{2} g_{\mu \nu} R^{p} \mathcal{F}(\square) R^{q}+\left(R_{\mu \nu} \Phi-K_{\mu \nu} \Phi\right) \\
& +\frac{1}{2} \sum_{n=1}^{\infty} f_{n} \sum_{l=0}^{n-1}\left(g_{\mu \nu} \partial^{\alpha} \square^{l} R^{p} \partial_{\alpha} \square^{n-1-l} R^{q}-2 \partial_{\mu} \square^{l} R^{p} \partial_{\nu} \square^{n-1-l} R^{q}+g_{\mu \nu} \square^{l} R^{p} \square^{n-l} R^{q}\right)=0,
\end{aligned}
$$

where $\Phi=p R^{p-1} \mathcal{F}(\square) R^{q}+q R^{q-1} \mathcal{F}(\square) R^{p}$.

Corollary 3.2. For $\mathcal{H}(R)=R^{p}$ and $\mathcal{G}(R)=R^{q}$ the equations of motion (36) are equivalent to the following two equations:

$$
\frac{1}{16 \pi G}(4 \Lambda-R)-2 R^{p} \mathcal{F}(\square) R^{q}+(R \Phi+3 \square \Phi)+\sum_{n=1}^{\infty} f_{n} \sum_{l=0}^{n-1}\left(\partial^{\mu} \square^{l} R^{p} \partial_{\mu} \square^{n-1-l} R^{q}+2 \square^{l} R^{p} \square^{n-l} R^{q}\right)=0,
$$




$$
\begin{aligned}
& \frac{1}{16 \pi G}\left(G_{00}+\Lambda g_{00}\right)-\frac{1}{2} g_{00} R^{p} \mathcal{F}(\square) R^{q}+\left(R_{00} \Phi-K_{00} \Phi\right) \\
& +\frac{1}{2} \sum_{n=1}^{\infty} f_{n} \sum_{l=0}^{n-1}\left(g_{00} \partial^{\alpha} \square^{l} R^{p} \partial_{\alpha} \square^{n-1-l} R^{q}+g_{00} \square^{l} R^{p} \square^{n-l} R^{q}-2 \partial_{0} \square^{l} R^{p} \partial_{0} \square^{n-1-l} R^{q}\right)=0 .
\end{aligned}
$$

\section{Constant scalar curvature}

Theorem 4.1. Let $R=R_{0}=$ constant. Then, solution of equations of motion (36) has the form

1. For $R_{0}>0, a(t)=\sqrt{\frac{6 k}{R_{0}}+\sigma e^{\sqrt{\frac{R_{0}}{3}} t}+\tau e^{-\sqrt{\frac{R_{0}}{3}}} t}$, where $9 k^{2}=R_{0}^{2} \sigma \tau, \sigma, \tau \in \mathbb{R}$.

2. For $R_{0}=0, a(t)=\sqrt{-k t^{2}+\sigma t+\tau}$, where $\sigma^{2}+4 k \tau=0, \sigma, \tau \in \mathbb{R}$

3. For $R_{0}<0, a(t)=\sqrt{\frac{6 k}{R_{0}}+\sigma \cos \sqrt{\frac{-R_{0}}{3}} t+\tau \sin \sqrt{\frac{-R_{0}}{3}}}$, where $36 k^{2}=R_{0}^{2}\left(\sigma^{2}+\tau^{2}\right), \sigma, \tau \in \mathbb{R}$, where $k$ is curvature parameter.

Proof. Since $R=R_{0}$ one has

$$
6\left(\frac{\ddot{a}}{a}+\left(\frac{\dot{a}}{a}\right)^{2}+\frac{k}{a^{2}}\right)=R_{0}
$$
cients

The change of variable $b(t)=a^{2}(t)$ yields second order linear differential equation with constant coeffi-

$$
3 \ddot{b}-R_{0} b=-6 k
$$

Depending on the sign of $R_{0}$ we have the following solutions for $b(t)$

$$
\begin{array}{ll}
R_{0}>0, & b(t)=\frac{6 k}{R_{0}}+\sigma e^{\sqrt{\frac{R_{0}}{3}} t}+\tau e^{-\sqrt{\frac{R_{0}}{3}} t}, \\
R_{0}=0, & b(t)=-k t^{2}+\sigma t+\tau, \\
R_{0}<0, & b(t)=\frac{6 k}{R_{0}}+\sigma \cos \sqrt{\frac{-R_{0}}{3}} t+\tau \sin \sqrt{\frac{-R_{0}}{3}} t .
\end{array}
$$

Putting $R=R_{0}=$ const into $(37)$ and $(\underline{38})$ one obtains the following two equations:

$$
f_{0} R_{0}^{p+q}(p+q-2)=\frac{R_{0}-4 \Lambda}{16 \pi G}, \quad f_{0} R_{0}^{p+q-1}\left(\frac{1}{2} R_{0}+(p+q) R_{00}\right)=\frac{-G_{00}+\Lambda}{16 \pi G} .
$$

Equations (42) will have a solution if and only if

$$
R_{0}^{p+q-1}\left(R_{0}+4 R_{00}\right)\left(R_{0}+\left(2 \Lambda-R_{0}\right)(p+q)\right)=0 .
$$

In the first case we take $R_{0}+4 R_{00}=0$ that yields the following conditions on the parameters $\sigma$ and $\tau$ :

$$
\begin{array}{ll}
R_{0}>0, & 9 k^{2}=R_{0}^{2} \sigma \tau, \\
R_{0}=0, & \sigma^{2}+4 k \tau=0, \\
R_{0}<0, & 36 k^{2}=R_{0}^{2}\left(\sigma^{2}+\tau^{2}\right) .
\end{array}
$$


Solutions given in (41) together with conditions (44) restrict the possibilities for the parameter $k$.

Theorem 4.2. 1. If $R_{0}>0$ then for $k=0$ there is solution with constant Hubble parameter, for $k=+1$ the solution is $a(t)=\sqrt{\frac{12}{R_{0}}} \cosh \frac{1}{2}\left(\sqrt{\frac{R_{0}}{3}} t+\varphi\right)$ and for $k=-1$ it is $a(t)=\sqrt{\frac{12}{R_{0}}} \mid \sinh \frac{1}{2}\left(\sqrt{\frac{R_{0}}{3}} t+\varphi\right)$, where $\sigma+\tau=\frac{6}{R_{0}} \cosh \varphi$ and $\sigma-\tau=\frac{6}{R_{0}} \sinh \varphi$.

2. If $R_{0}=0$ then for $k=0$ the solution is $a(t)=\sqrt{\tau}=$ const and for $k=-1$ the solution is $a(t)=\left|t+\frac{\sigma}{2}\right|$.

3. If $R_{0}<0$ then for $k=-1$ the solution is $a(t)=\sqrt{\frac{-12}{R_{0}}}\left|\cos \frac{1}{2}\left(\sqrt{-\frac{R_{0}}{3}} t-\varphi\right)\right|$, where $\sigma=\frac{-6}{R_{0}} \cos \varphi$ and $\tau=\frac{-6}{R_{0}} \sin \varphi$.

Proof. Let $R_{0}>0$. Set $k=0$ then we obtain solution with constant Hubble parameter. Alternatively, if we set $k=+1$ then there is $\varphi$ such that $\sigma+\tau=\frac{6}{R_{0}} \cosh \varphi$ and $\sigma-\tau=\frac{6}{R_{0}} \sinh \varphi$. Moreover, we obtain

$$
b(t)=\frac{12}{R_{0}} \cosh ^{2} \frac{1}{2}\left(\sqrt{\frac{R_{0}}{3}} t+\varphi\right), \quad a(t)=\sqrt{\frac{12}{R_{0}}} \cosh \frac{1}{2}\left(\sqrt{\frac{R_{0}}{3}} t+\varphi\right) .
$$

At the end, if we set $k=-1$ one can transform $b(t)$ to

$$
b(t)=\frac{12}{R_{0}} \sinh ^{2} \frac{1}{2}\left(\sqrt{\frac{R_{0}}{3}} t+\varphi\right), \quad a(t)=\sqrt{\frac{12}{R_{0}}} \mid \sinh \frac{1}{2}\left(\sqrt{\frac{R_{0}}{3}} t+\varphi\right) .
$$

Let $R_{0}=0$. If $k=0$ then function $b(t)$ and consequently $a(t)$ become constants which leads to a solution $a(t)=\sqrt{\tau}=$ const. On the other hand if $k \neq 0$ then we can write

$$
b(t)=-k\left(t-\frac{\sigma}{2 k}\right)^{2} .
$$

If $k=+1$ then there are no solutions for the scale factor $a(t)$, because $b(t) \leq 0$. On the other hand, when $k=-1$ the scalar factor becomes

$$
a(t)=\left|t+\frac{\sigma}{2}\right| \text {. }
$$

In the last case, let $R_{0}<0$. If $k=-1$ we can find $\varphi$ such that $\sigma=\frac{-6}{R_{0}} \cos \varphi$ and $\tau=\frac{-6}{R_{0}} \sin \varphi$ and rewrite $a(t)$ and $b(t)$ as

$$
b(t)=\frac{-12}{R_{0}} \cos ^{2} \frac{1}{2}\left(\sqrt{-\frac{R_{0}}{3}} t-\varphi\right), \quad a(t)=\sqrt{\frac{-12}{R_{0}}} \mid \cos \frac{1}{2}\left(\sqrt{-\frac{R_{0}}{3}} t-\varphi\right) .
$$

In the case $k=+1$ one can transform $b(t)$ to $b(t)=\frac{12}{R_{0}} \sin ^{2} \frac{1}{2}\left(\sqrt{-\frac{R_{0}}{3}} t-\varphi\right)$, which is non positive and hence yields no solutions.

Theorem 4.3. If in (43) we take

$$
R_{0}^{p+q-1}\left(R_{0}+(p+q)\left(2 \Lambda-R_{0}\right)\right)=0
$$

then:

1. For $p+q \geq 1$ there is obvious solution $R_{0}=0$. In particular if $p+q=1$ then (50) is satisfied for any $R_{0} \neq 0$ if $\Lambda=0$.

2. For $p+q=0$ there is no solution.

3. For $p+q \neq 0,1$ there is a unique value $R_{0}=\frac{2 \Lambda(p+q)}{p+q-1}$ that gives a solution. Since $p$ and $q$ are integers the value of $R_{0}$ in the last equation is always positive, and for $k=0$ the solution $b(t)$ is a linear combination of exponential functions.

Proof of this theorem is evident. 


\section{References}

[1] T. Clifton, P. G. Ferreira, A. Padilla, C. Skordis, "Modified gravity and cosmology", Physics Reports 513 (2012) 1-189; arXiv:1106.2476v2 [astro-ph.CO]].

[2] S. Nojiri, S. D. Odintsov, "Unified cosmic history in modified gravity: from $F(R)$ theory to Lorentz non-invariant models," Physics Reports 505 (2011) 59-144; |arXiv:1011.0544v4 [gr-qc]].

[3] R. P. Woodard, "Nonlocal models of cosmic acceleration," arXiv:1401.0254 [astro-ph.CO]] (2014).

[4] B. Dragovich, "Nonlocal modified gravity and cosmology," to be published in Springer Proceedings in Mathematics \& Statistics.

[5] T. Biswas, A. Mazumdar, W. Siegel, "Bouncing universes in string-inspired gravity," Journal of Cosmology and Astroparticle Physics 0603 (2006) 009; |arXiv:hep-th/0508194].

[6] T. Biswas, T. Koivisto, A. Mazumdar, "Towards a resolution of the cosmological singularity in non-local higher derivative theories of gravity," Journal of Cosmology and Astroparticle Physics 1011 (2010) 008; arXiv:1005.0590v2 [hep-th]].

[7] T. Biswas, E. Gerwick, T. Koivisto, A. Mazumdar, "Towards singularity and ghost free theories of gravity", Physical Review Letters 108 (2012) 031101; [arXiv:1110.5249v2 [gr-qc]].

[8] T. Biswas, A. S. Koshelev, A. Mazumdar, S. Yu. Vernov, "Stable bounce and inflation in non-local higher derivative cosmology", Journal of Cosmology and Astroparticle Physics 08 (2012) 024; arXiv:1206.6374 [astro-ph.CO]].

[9] A. S. Koshelev, S. Yu. Vernov, "On bouncing solutions in non-local gravity," arXiv:1202.1289v1 [hep-th]].

[10] I. Dimitrijevic, B. Dragovich, J. Grujic, Z. Rakic, "On modified gravity," Springer Proceedings in Mathematics \& Statistics 36 (2013) 251-259; [arXiv:1202.2352 [hep-th]].

[11] I. Dimitrijevic, B. Dragovich, J. Grujic, Z. Rakic, “New cosmological solutions in nonlocal modified gravity,” Romanian Journal of Physics 58 (5-6) (2013) 550-559; arXiv:1302.2794 [gr-qc]].

[12] T. Biswas, A. Conroy, A. S. Koshelev, A. Mazumdar, "Generalized gost-free quadratic curvature gravity", arXiv:1308.2319 [hep-th]] (2013).

[13] I. Dimitrijevic, B. Dragovich, J. Grujic, Z. Rakic, "A new model of nonlocal modified gravity," Publications de l'Institut Mathematique 94 (108) (2013) 187-196; arXiv:1411.5034[hep-th]]. 